\title{
Quantum Theory of Fields and Properties of Quantum Systems
}

\author{
Fernand Tshizanga Mpinga \\ Department of Mechanics, Superior Institute of Applied Techniques, Kinshasa, Democratic Republic of Congo
}

Email address:

tshizafer@hotmail.com

\section{To cite this article:}

Fernand Tshizanga Mpinga. Quantum Theory of Fields and Properties of Quantum Systems. American Journal of Physics and Applications. Vol. 7, No. 4, 2019, pp. 93-100. doi: 10.11648/j.ajpa.20190704.11

Received: April 27, 2019; Accepted: June 3, 2019; Published: July 4, 2019

\begin{abstract}
Quantum theory of fields is the most general theory to date. It has an extremely wide field of application: - the physics of elementary particles and their interactions (summarized by the Standard Model); - the physics of the universe close to the Big Bang (primordial fluctuations from which the formation of the structure of the universe originates, evaporation of black holes, Hawking radiation); - the formalism of condensed matter physics, with applications such as superconductivity, superfluidity, phase transitions. Indeed, the quantum theory of fields has been successfully implemented in quantum systems, notably in research on fundamental state energy, elementary excitations spectrum, degeneracy parameters: long range order, Bogoliubov approximation, density matrix diagonalization,..., as well as the characteristics of these systems: movement equation, dynamics of the system,.... Three different quantum systems were concerned in this theoretical study: - a gas of identical atoms of spin zero confined into the trap; - electron gas of spin $1 / 2$ into the metal; - and a gathering of identical ions of spin zero at high density confined into a radiofrequency linear Paul trap. The microscopic theory was used in the each case and the results obtained by the researchers are presented.
\end{abstract}

Keywords: Quantum Theory of Fields, Quantum Systems, Microscopic Theory, Bosons, Fermions, Second Quantization, Density Matrix, Fundamental State Energy

\section{Introduction}

In quantum theory of fields, particles are described by the fields; the quanta of these fields being particles [1]. This theory, also used for the study of elementary particles, has been successfully used within the framework of quantum systems, especially in the case of the spin zero boson system without electric charge confined in a magneto-optical trap [2,3], and electron gas confined in a metal [4]. The issue at stake in this paper is to remind the definition of quantum systems, to present some theoretical results obtained on using this theory in the study of neutral boson gas confined in a magneto-optical trap, and electron gas in a metal. The paper will end by giving our results, on the study of an ultra-cold and high density non-neutral plasma of identical spin zero ions confined in a radiofrequency linear Paul trap [5].

\section{Dogmas of Quantum Theory of Fields}

The key element of quantum theory of fields is the pooling of quantum theory and the theory of special relativity [6]. Einstein's special relativity in demonstrating that mass and energy can be converted into one another has put an end to matter- energy dualism. The wave-particle duality has been abolished by the new quantum theory, where field and particles no longer appear as distinct, but as complementary. The essential stage of this latest discovery is the article published in 1928 by Jordan and Eugene Wigner, and the two articles published in 1929-30 by Heisenberg and Pauli; in these articles, it is shown that material particles can be considered as the quantons of various fields in the same way that the photon is the quantum of the electromagnetic field, each type of elementary particle being supposed to correspond to a field $[1,7]$. The dogmas of the quantum theory of fields can be summed up in five points: - The essential material reality is a set of fields;

The fields obey the principles of special relativity and quantum theory;

The intensity of a field at a particular point gives the probability of finding the quantons associated with it, that is 
to say, the fundamental particles observed by experimenters;

The fields interact with each other, which means that the quantons associated with them interact, these interactions are themselves mediated by quantons;

There is nothing outside of that [7].

These points form the basic image of physics and the task of physicists is to bring all the rest of physics into this strict framework.

\section{Quantum Theory of Fields Really Necessary}

Whenever we have a system with an interacting body, of which microscopic physics is supposed to be known, it is described by an effective theory which is a renormalizable theory of fields [8]. This results in an extremely wide field of application which is articulated around three main areas:

The physics of elementary particles and their interactions, summarized by the Standard Model;

The formalism of condensed matter physics, with applications such as superconductivity, superfluidity, phase transitions;

The physics of the universe close to the Big Bang: primordial fluctuations from which the formation of the structure of the universe originates, evaporation of black holes (Hawking radiation), etc. [6].

Quantum theory of fields has given rise to new ideas: antimatter, vacuum physics, identical particles, and exchange forces that have transformed the physicists view reality. The development of this theory has trained physicists in new areas of mathematics, such as Hilbert spaces, operator theory and matrix algebra [7].

\section{Quantum Systems}

Quantum system is obtained by confining identical particles (molecules, atoms, electrons, ions...) in a box or a trap. When the density of the particles is very high or if the temperature of the system is lower, the quantum effects of these particles show up [9]. The density of molecules in air room temperature and atmospheric pressure is about $10^{19} \mathrm{~cm}^{-}$ 3. In liquids and solids, the density of atoms is of order of $10^{22} \mathrm{~cm}^{-3}$, while the density of nucleons in atom is about $10^{38}$ $\mathrm{cm}^{-3}$. At room temperature, electrons in metal (about $10^{22}$ to $10^{23}$ electrons per $\mathrm{cm}^{-3}$ ) are in a quantum system. In solids, quantum effects become strong for electrons in metals below the Fermi temperature, which is typically $10^{4}-10^{5} \mathrm{~K}$. For helium liquids, the temperature required for observing quantum phenomena is of order of $1 \mathrm{~K}$. Due to the much higher particle density in atomic nuclei, the corresponding degeneracy temperature is about $10^{11} \mathrm{~K}[10]$.

Classical mechanics as well as quantum mechanics are no longer used. Instead, we have recourse to quantum statistical physics as well as to quantum theory of fields. So, entire spin particles (bosons) and half-entire spin particles (fermions) appear. Bosons comply with Bose-Einstein statistics whereas fermions comply with Fermi-Dirac statistics. Microscopic theory has allowed to study the characteristics of these quantum systems by using fields corresponding to imperceptible identical particles of each system [11].

\section{Properties of Quantum Systems}

For the quantum system, being made of indiscernable and identical particles, the use of the second quantization method offers a more flexible representation that is more concised and neater. The second quantization usually considers a group of particles more or less independent paired by weak interactions [4]. Particle operators which are concerned with second quantization are attributable to the quantum theory of fields [12].

\subsection{Microscopic Theory}

The microscopic theory allows to inquire the properties of the groups of identical particles enclosed in a box or a trap[9]. The points of microscopic theory are: - Particle field, in quantum theory fields stand for Klein-Gordon solutions deriving from a free Lagrangian density which is an invariant of Lorentz admitting a positive Hamiltonian density. Particle operator (boson operator, fermion operator) annihiles or creates one-particle. - Space of system quantum states or Fock space. - Field operators of annihilation and creation of particle of impulsion $\vec{p}$ at the position $\vec{r}$. - Bogoliubov prescription for a system of particles in the fundamental state with interaction. - Density matrix of the system, it serves to characterize the correlations between particles located at $\vec{r}$ and $\vec{r}$ '. - Hamiltonian of the second quantization of particle system with short or long range interaction. - Temporal evolution of the Heisenberg field operator allowing to establish the Gross-Pitaevskii equation (neutral bosons with short range interaction) or Hartree-Fock equation (system with long range interaction). - Energy calculation of the fundamental state. - Spectrum of elementary excitations...[13].

\subsection{Properties of Identical Neutral Particles of Spin Zero (Atoms, Molecules) in Trap or Box}

The application of microscopic theory to the system of bosons in interaction is but satisfied when there exists a weak repulsive interaction between particles, or a gas diluated with arbitrary repulsive interaction between particles [9]. BoseEinstein condensation (BEC) is a very active research field in condensed matter physics and material science. In the laboratory a suitable example of BEC is provided by a gas of an appropriate density of alkali-metal atoms. All stable alkali species $\mathrm{Li}, \mathrm{Na}, \mathrm{K}, \mathrm{Rb}$, and $\mathrm{Cs}$ have been condensed $[16,17,18]$. Here are the results of the application of microscopic theory to the system of bosons of spin zero, with Wander's short range interaction, confined $[9,10,14]$ :

\subsubsection{Real Scalar Field}

$$
\varphi(\mathrm{x})=\frac{1}{\sqrt{(2 \pi \hbar)^{3}}} \int \frac{\mathrm{d}^{3} \mathrm{k}}{\sqrt{2 \omega_{\mathrm{k}}}}\left[\mathrm{a}_{\overrightarrow{\mathrm{k}}}^{-} \mathrm{e}^{-\mathrm{ikx}}+\mathrm{a}_{\overrightarrow{\mathrm{k}}}^{+} \mathrm{e}^{\mathrm{ikx}}\right]
$$

Equation (1) is the expansion of Klein-Gordon solution, 
which derives to Lagrangian density of zero spin particle with the mass $\mathrm{m}$.

\subsubsection{Boson Operators (One-boson Annihilation/Creation Operators)}

$$
\hat{\mathrm{a}}_{\mathrm{k}}^{ \pm}=\frac{\mp \mathrm{i}}{\sqrt{(2 \pi)^{3}}} \int \frac{\mathrm{d}^{3} \mathrm{x}}{\sqrt{2 \omega_{\mathrm{k}}}}\left[\widehat{\Pi}(\mathrm{x}) \pm \mathrm{ik}_{0} \widehat{\varphi}(\mathrm{x})\right] \mathrm{e}^{\mp \mathrm{ikx}}
$$

Operators (2) are reducible to operators $\widehat{\Pi}(\mathrm{x})$ and $\widehat{\varphi}(\mathrm{x})$ of the quantum system.

\subsubsection{Commutation Relations of Boson Operators}

$$
\left\{\begin{array}{c}
{\left[\hat{\mathrm{a}}_{\overrightarrow{\mathrm{k}}}, \hat{\mathrm{a}}_{\overrightarrow{\mathrm{k}} \prime}^{\dagger}\right]=\delta_{\overrightarrow{\mathrm{k}} \overrightarrow{\mathrm{k}}^{\prime}}} \\
{\left[\hat{\mathrm{a}}_{\overrightarrow{\mathrm{k}}}, \hat{\mathrm{a}}_{\overrightarrow{\mathrm{k}} \prime}\right]=\left[\hat{\mathrm{a}}_{\overrightarrow{\mathrm{k}}}^{\dagger}, \hat{\mathrm{a}}_{\overrightarrow{\mathrm{k}} \prime}^{\dagger}\right]=0}
\end{array}\right.
$$

\subsubsection{Fock Space of Quantum States}

$$
\left\{\begin{array}{c}
|\mathrm{n}\rangle=\frac{\left(\hat{\mathrm{a}}^{\dagger}\right)^{\mathrm{n}}}{\sqrt{\mathrm{n} !}}|0\rangle \mathrm{n}=1,2, \ldots \\
\langle 0 \mid 0\rangle=1 \\
\langle\mathrm{~m} \mid \mathrm{n}\rangle=\delta_{\mathrm{mn}}
\end{array}\right.
$$

\subsubsection{Field Operators of Annihilation and Creation of Boson with Impulsion $\vec{p}$ at the Position $\vec{r}$}

$$
\left\{\begin{array}{c}
\widehat{\psi}(\overrightarrow{\mathrm{r}}) \equiv \sum_{\overrightarrow{\mathrm{p}}} \hat{\mathrm{a}}_{\overrightarrow{\mathrm{p}}}\langle\mathrm{r} \mid \mathrm{p}\rangle=\sum_{\overrightarrow{\mathrm{p}}} \hat{\mathrm{a}}_{\overrightarrow{\mathrm{p}}} \varphi_{\overrightarrow{\mathrm{p}}}(\overrightarrow{\mathrm{r}})=\frac{1}{\sqrt{\Omega}} \sum_{\vec{p}} \hat{\mathrm{a}}_{\overrightarrow{\mathrm{p}}} e^{i \vec{p} \cdot \vec{r}} \\
\widehat{\Psi}^{\dagger}(\overrightarrow{\mathrm{r}}) \equiv \sum_{\overrightarrow{\mathrm{p}}} \hat{\mathrm{a}}_{\overrightarrow{\mathrm{p}}}^{\dagger}\langle\mathrm{p} \mid \mathrm{r}\rangle=\sum_{\overrightarrow{\mathrm{p}}} \hat{\mathrm{a}}_{\overrightarrow{\mathrm{p}}}^{\dagger} \varphi_{\overrightarrow{\mathrm{p}}}^{*}(\overrightarrow{\mathrm{r}})=\frac{1}{\sqrt{\Omega}} \sum_{\vec{p}} \hat{\mathrm{a}}_{\overrightarrow{\mathrm{p}}}^{\dagger} e^{-i \vec{p} \cdot \vec{r}}
\end{array}\right.
$$

\subsubsection{Properties According to Field Operators}

The commutation relations of field operators have the form:

$$
\left\{\begin{array}{c}
{\left[\widehat{\psi}(\overrightarrow{\mathrm{r}}), \widehat{\psi}^{\dagger}\left(\overrightarrow{\mathrm{r}}^{\prime}\right)\right]=\delta\left(\overrightarrow{\mathrm{r}}-\overrightarrow{\mathrm{r}}^{\prime}\right)} \\
{\left[\widehat{\psi}(\overrightarrow{\mathrm{r}}), \widehat{\psi}\left(\overrightarrow{\mathrm{r}}^{\prime}\right)\right]=\left[\widehat{\psi}^{\dagger}(\overrightarrow{\mathrm{r}}), \widehat{\psi}^{\dagger}\left(\overrightarrow{\mathrm{r}}^{\prime}\right)\right]=0}
\end{array}\right.
$$

The density matrix of the system, which serves to

$$
\int \mathrm{d} \overrightarrow{\mathrm{r}}^{\prime} \rho_{1}\left(\overrightarrow{\mathrm{r}}^{\prime}, \overrightarrow{\mathrm{r}}\right) \varphi_{\overrightarrow{\mathrm{p}}}\left(\overrightarrow{\mathrm{r}}^{\prime}\right)=\int \mathrm{d} \overrightarrow{\mathrm{r}}^{\prime} \frac{1}{\Omega} \sum_{\overrightarrow{\mathrm{p}}} N_{\vec{p}} \mathrm{e}^{i \overrightarrow{\mathrm{p}} \cdot\left(\overrightarrow{\mathrm{r}}-\overrightarrow{\mathrm{r}}^{\prime}\right)} \varphi_{\overrightarrow{\mathrm{p}}}\left(\overrightarrow{\mathrm{r}}^{\prime}\right)=\int \mathrm{d} \overrightarrow{\mathrm{r}}^{\prime} \frac{1}{(2 \pi)^{3}} \int \mathrm{d} \overrightarrow{\mathrm{p}} \mathrm{e}^{\mathrm{i} \overrightarrow{\mathrm{p}} \cdot(\overrightarrow{\mathrm{r}}-\overrightarrow{\mathrm{r}} \prime)} N_{\vec{p}} \varphi_{\overrightarrow{\mathrm{p}}}\left(\overrightarrow{\mathrm{r}}^{\prime}\right)=\int \mathrm{d} \overrightarrow{\mathrm{r}}^{\prime} \delta\left(\overrightarrow{\mathrm{r}}-\overrightarrow{\mathrm{r}}^{\prime}\right) N_{\vec{p}} \varphi_{\overrightarrow{\mathrm{p}}}\left(\overrightarrow{\mathrm{r}}^{\prime}\right)=\mathrm{N}_{\overrightarrow{\mathrm{p}}} \varphi_{\overrightarrow{\mathrm{p}}}(\overrightarrow{\mathrm{r}})
$$

It corresponds to the eigenfunction $\varphi_{\vec{p}}(\vec{r})$ with the largest eigenvalue $\mathrm{N}_{\overrightarrow{\mathrm{p}}}$. This procedure has been used, for example, to explore Bose-Einstein condensation in finite drops of liquid helium by Lewart, Pandharipande, and Pieper (1988).

Bose-Einstein condensation occurs when the number of atoms $\mathrm{N}_{0}$ of a particular single-particle state becomes very large: $\mathrm{N}_{0} \gg 1$ and the ratio $\mathrm{N}_{0} / \mathrm{N}$ remains finite in thermodynamic limit $\mathrm{N} \rightarrow \infty$. In this limit the states with $\mathrm{N}_{0}$ and $N_{0} \pm 1 \approx N_{0}$ correspond to the same physical configuration and, consequently, the operators $\hat{\mathrm{a}}_{0}$ and $\hat{\mathrm{a}}_{0}^{\dagger}$ can be treated like c numbers: $\hat{\mathrm{a}}_{0}=\hat{\mathrm{a}}_{0}^{\dagger}=\sqrt{\mathrm{N}_{0}}$. BEC occurs, for a uniform gas, in the single-particle state $\psi_{0}=\frac{1}{\sqrt{\mathrm{V}}}$ having zero momentum, and the field operator $\widehat{\psi}(\overrightarrow{\mathrm{r}})$ can then be decomposed in the form

$$
\widehat{\psi}(\overrightarrow{\mathrm{r}})=\sqrt{\mathrm{N}_{0} / \mathrm{V}}+\widehat{\psi}^{\prime}(\overrightarrow{\mathrm{r}})
$$

characterize the correlations between particles located at $\vec{r}$ and $\vec{r}^{\prime}$, is defined by

$$
\rho\left(\overrightarrow{\mathrm{r}}, \overrightarrow{\mathrm{r}}^{\prime}\right)=\left\langle\varphi(\mathrm{N})\left|\widehat{\Psi}^{\dagger}(\overrightarrow{\mathrm{r}}) \widehat{\psi}\left(\overrightarrow{\mathrm{r}}^{\prime}\right)\right| \varphi(\mathrm{N})\right\rangle=\sum_{\overrightarrow{\mathrm{p}}} \mathrm{N}_{\overrightarrow{\mathrm{p}}} \varphi_{\overrightarrow{\mathrm{p}}}^{*}(\overrightarrow{\mathrm{r}}) \varphi_{\overrightarrow{\mathrm{p}}}\left(\overrightarrow{\mathrm{r}}^{\prime}\right),
$$

it leads to the long range order. Let's consider the density matrix in the ground state

$$
\rho_{0}\left(\overrightarrow{\mathrm{r}}, \overrightarrow{\mathrm{r}}^{\prime}\right)=\mathrm{N}_{0} \varphi_{0}^{*}(\overrightarrow{\mathrm{r}}) \varphi_{0}\left(\overrightarrow{\mathrm{r}}^{\prime}\right)+\sum_{\overrightarrow{\mathrm{p}} \neq 0} \mathrm{~N}_{\overrightarrow{\mathrm{p}}} \varphi_{\overrightarrow{\mathrm{p}}}^{*}(\overrightarrow{\mathrm{r}}) \varphi_{\overrightarrow{\mathrm{p}}}\left(\overrightarrow{\mathrm{r}}^{\prime}\right)
$$

The first term at right-hand side is of infinite range in $\left(\vec{r}-\vec{r}^{\prime}\right)$, it arises directly as a consequence of the macroscopic occupation of a single quantum state, the condensate.

$$
\lim _{\left|\vec{r}^{\prime}-\vec{r}\right| \rightarrow \infty} \rho_{0}\left(\vec{r}, \vec{r}^{\prime}\right)=\frac{N_{O}}{\Omega}
$$

The second term $\sum_{\overrightarrow{\mathrm{p}} \neq 0} \mathrm{~N}_{\overrightarrow{\mathrm{p}}} \varphi_{\overrightarrow{\mathrm{p}}}^{*}(\overrightarrow{\mathrm{r}}) \varphi_{\overrightarrow{\mathrm{p}}}\left(\overrightarrow{\mathrm{r}}^{\prime}\right)$ has a finite range $\sum_{\vec{p} \neq 0} N_{\vec{p}}$, it describes local correlations between the excited system particles.

$$
\lim _{\left|\overrightarrow{\mathrm{r}}^{\prime}-\overrightarrow{\mathrm{r}}\right| \rightarrow 0} \rho_{0}\left(\overrightarrow{\mathrm{r}}, \overrightarrow{\mathrm{r}}^{\prime}\right)=\frac{1}{\Omega}\left(\mathrm{N}_{\mathrm{O}}+\sum_{\overrightarrow{\mathrm{p}} \neq 0} \mathrm{~N}_{\overrightarrow{\mathrm{p}}}\right)
$$

The presence of a term with an infinite range may be considered as characteristic of a superfluid boson system [9].

In finite- sized system neither the concept of broken gauge symmetry, nor the one of off-diagonal long-range order can be applied. The condensate wave function $\Phi$ nevertheless still has a clear meaning: it can in fact be determined through the diagonalization of the one-body density matrix [14],

$$
\rho_{1}\left(\overrightarrow{\mathrm{r}}^{\prime}, \overrightarrow{\mathrm{r}}\right)=\left\langle\widehat{\psi}^{\dagger}\left(\overrightarrow{\mathrm{r}}^{\prime}\right) \widehat{\psi}(\overrightarrow{\mathrm{r}})\right\rangle
$$

The diagonalization of this one-body density matrix is

By treating the operator $\widehat{\psi}(\overrightarrow{\mathrm{r}})$ as a small perturbation, Bogoliubov developed the "first-order" theory for the excitations of interacting Bose-gas. The generalization of the Bogoliubov prescription to the case of non-uniform and timedependent configurations is given by

$$
\begin{array}{r}
\widehat{\psi}(\overrightarrow{\mathrm{r}}, \mathrm{t})=\sqrt{\mathrm{N}_{0}} \varphi_{\mathrm{o}}(\overrightarrow{\mathrm{r}}, \mathrm{t})+\sum_{\delta \overrightarrow{\mathrm{p}} \neq 0} \hat{\mathrm{a}}_{\overrightarrow{\mathrm{p}}} \varphi_{\overrightarrow{\mathrm{p}}}(\overrightarrow{\mathrm{r}}, \mathrm{t})=\phi(\overrightarrow{\mathrm{r}}, \mathrm{t})+ \\
(12)
\end{array}
$$

Where one has used the Heisenberg representation for the field operators [9]. The function $\phi(\overrightarrow{\mathrm{r}}, \mathrm{t})=\sqrt{\mathrm{N}_{0}} \varphi_{\mathrm{o}}(\overrightarrow{\mathrm{r}}, \mathrm{t})$ is a classical field having the meaning of an order parameter and is often called the "wave-function of the condensate".

\subsubsection{Properties According to Hamiltonian Operator}

The many-body Hamiltonian describing $\mathrm{N}$ interacting bosons confined by an external potential is given, in second quantization by: 


$$
\widehat{H}=\int d \vec{r} \widehat{\psi}^{\dagger}(\vec{r})\left[\frac{\widehat{p}^{2}}{2 m}+\widehat{V}_{\text {piège }}\right] \widehat{\psi}(\vec{r})+\frac{1}{2} \int d \vec{r} \widehat{U}_{0} \widehat{\psi}^{\dagger}(\vec{r}) \widehat{\psi}^{\dagger}(\vec{r}) \widehat{\psi}(\vec{r}) \widehat{\psi}(\vec{r})
$$

where $\widehat{\psi}^{\dagger}(\overrightarrow{\mathrm{r}})$ and $\widehat{\psi}(\overrightarrow{\mathrm{r}})$ are the boson field operators that create and annihilate the particle at the point $\vec{r}$, respectively, and $\widehat{\mathrm{U}}_{0}$ is the two-body interatomic potential. The ground state of the system, as well as its thermodynamic properties, can be directly calculated starting from this Hamiltonian[14].

In order to derive the equation for the condensate wave function $\phi(\vec{r}, t)$, one has to write the time evolution of the field operator $\widehat{\psi}(\vec{r}, t)$, using the Heisenberg equation with the many-body Hamiltonian (13)

$$
\mathrm{i} \hbar \frac{\partial \widehat{\Psi}(\vec{r}, \mathrm{t})}{\partial \mathrm{t}}=\left[-\frac{\hbar^{2}}{2 \mathrm{~m}} \widehat{\nabla}^{2}+\widehat{V}_{\text {piège }}+\widehat{\mathrm{U}}_{0} \widehat{\psi}^{\dagger}(\overrightarrow{\mathrm{r}}, \mathrm{t}) \widehat{\psi}(\overrightarrow{\mathrm{r}}, \mathrm{t})\right] \widehat{\psi}(\overrightarrow{\mathrm{r}}, \mathrm{t})
$$

When one replaces the operator $\widehat{\psi}(\vec{r}, t)$ with the classical field $\phi(r)$, equation (14) becomes

$$
\mathrm{i} \hbar \frac{\partial \phi_{0}(\overrightarrow{\mathrm{r}}, \mathrm{t})}{\partial \mathrm{t}}=\left[-\frac{\hbar^{2}}{2 \mathrm{~m}} \nabla^{2}+\mathrm{V}_{\text {piège }}+\mathrm{U}_{0} \eta_{0}(\overrightarrow{\mathrm{r}}, \mathrm{t})\right] \phi_{0}(\overrightarrow{\mathrm{r}}, \mathrm{t})
$$

$U_{0}$ is the effective potential. The use of this potential is compatible with the replacement of $\widehat{\psi}(\vec{r}, t)$ by $\phi(r)$. This equation, is known as Gross-Pitaevskii equation.

The fundamental state energy is given by

$$
\mathrm{E}_{0}=\langle 0|\widehat{\mathrm{H}}| 0\rangle \mathrm{E}_{0}=\sum_{\mathbf{p}} \varepsilon_{\mathbf{p}}^{0} \mathrm{~N}_{\mathbf{p}}+\frac{\mathrm{U}_{0}}{\Omega}\left(\mathrm{N}^{2}-\frac{\mathrm{N}_{0}^{2}}{2}\right)
$$

and the elementary excitations spectrum are

$$
\varepsilon_{\mathbf{p}}=\varepsilon_{\mathbf{p}}^{0}+\frac{\mathrm{N}}{\Omega} \mathrm{U}_{0}+\frac{\mathrm{N}-\mathrm{N}_{\mathbf{p}}}{\Omega} \mathrm{U}_{0}
$$

\subsection{Properties of High Density Electron Gas Confined in a Metal}

The electron gas, confined in a metal has been considered as a quantum gas to which microscopic theory has been applied $[4,9]$. The results of this inquiry are the following:

\subsubsection{Electron Field (Dirac Field)}

$$
\left\{\begin{array}{c}
\phi(\mathrm{x})=\frac{1}{\sqrt{\Omega}} \sum_{\mathrm{p}_{0}=\mathrm{E}_{\mathrm{p}}}\left(\frac{\mathrm{m}}{\mathrm{p}_{0}}\right)^{\frac{1}{2}} \sum_{\mathrm{r}=1}^{2}\left\{\mathrm{a}_{\mathrm{s}}^{(+)}(\overrightarrow{\mathrm{p}}) \mathrm{v}_{\mathrm{s}}(\overrightarrow{\mathrm{p}}) \mathrm{e}^{\mathrm{ipx}}+\mathrm{a}_{\mathrm{s}}^{(-)}(\overrightarrow{\mathrm{p}}) \mathrm{u}_{\mathrm{s}}(\overrightarrow{\mathrm{p}}) \mathrm{e}^{-\mathrm{ipx}}\right\} \\
\phi^{*}(\mathrm{x})=\frac{1}{\sqrt{\Omega}} \sum_{\mathrm{p}_{0}=\mathrm{E}_{\mathrm{p}}}\left(\frac{\mathrm{m}}{\mathrm{p}_{0}}\right)^{\frac{1}{2}} \sum_{\mathrm{r}=1}^{2}\left\{\mathrm{a}_{\mathrm{s}}^{*(+)}(\overrightarrow{\mathrm{p}}) \overline{\mathrm{u}}_{\mathrm{s}}(\overrightarrow{\mathrm{p}}) \mathrm{e}^{\mathrm{ipx}}+\mathrm{a}_{\mathrm{s}}^{*(-)}(\overrightarrow{\mathrm{p}}) \overline{\mathrm{v}}_{\mathrm{s}}(\overrightarrow{\mathrm{p}}) \mathrm{e}^{-\mathrm{ipx}}\right\}
\end{array}\right.
$$

\subsubsection{Electron and Positron Operators}

In order to get positron and electron operator, the fields are transformed into operators

$$
\left\{\begin{array}{c}
\widehat{\phi}(\mathrm{x})=\frac{1}{\sqrt{\Omega}} \sum_{\mathrm{p}_{0}=\mathrm{E}_{\mathrm{p}}}\left(\frac{\mathrm{m}}{\mathrm{p}_{0}}\right)^{\frac{1}{2}} \sum_{\mathrm{r}=1}^{2}\left(\hat{\mathrm{a}}_{\mathrm{s}}^{(+)}(\overrightarrow{\mathrm{p}}) \mathrm{v}_{\mathrm{s}}(\overrightarrow{\mathrm{p}}) \mathrm{e}^{\mathrm{ipx}}+\hat{\mathrm{a}}_{\mathrm{s}}^{(-)}(\overrightarrow{\mathrm{p}}) \mathrm{u}_{\mathrm{s}}(\overrightarrow{\mathrm{p}}) \mathrm{e}^{-\mathrm{ipx}}\right\} \\
\hat{\phi}^{\dagger}(\mathrm{x})=\frac{1}{\sqrt{\Omega}} \sum_{\mathrm{p}_{0}=\mathrm{E}_{\mathrm{p}}}\left(\frac{\mathrm{m}}{\mathrm{p}_{0}}\right)^{\frac{1}{2}} \sum_{\mathrm{r}=1}^{2}\left\{\hat{\mathrm{a}}_{\mathrm{s}}^{\dagger(+)}(\overrightarrow{\mathrm{p}}) \overline{\mathrm{u}}_{\mathrm{s}}(\overrightarrow{\mathrm{p}}) \mathrm{e}^{\mathrm{ipx}}+\hat{\mathrm{a}}_{\mathrm{s}}^{\dagger(-)}(\overrightarrow{\mathrm{p}}) \overline{\mathrm{v}}_{\mathrm{s}}(\overrightarrow{\mathrm{p}}) \mathrm{e}^{-\mathrm{ipx}}\right\}
\end{array}\right.
$$

The coefficients of the development (19) are determined by inverse Fourier transforms as:

$$
\left\{\begin{array}{c}
\hat{\mathrm{a}}_{\mathrm{s}}^{(-)}(\overrightarrow{\mathrm{p}})=\frac{1}{\sqrt{\Omega}}\left(\frac{\mathrm{m}}{\mathrm{p}_{0}}\right)^{\frac{1}{2}} \int \mathrm{d} \overrightarrow{\mathrm{x}} \mathrm{e}^{\mathrm{i} k \mathrm{x}} \mathrm{u}_{\mathrm{s}}(\mathrm{p}) \overleftrightarrow{\partial_{0}} \widehat{\phi}(\mathrm{x}) \text { (annihilation operator of fermion) } \\
\hat{\mathrm{a}}_{\mathrm{s}}^{(+)}(\overrightarrow{\mathrm{p}})=\frac{1}{\sqrt{\Omega}}\left(\frac{\mathrm{m}}{\mathrm{p}_{0}}\right)^{\frac{1}{2}} \int \mathrm{d} \overrightarrow{\mathrm{x}} \mathrm{e}^{\mathrm{i} \mathrm{kx}} \widehat{\phi}^{\dagger}(\mathrm{x}) \overleftrightarrow{\partial_{0}} \mathrm{v}_{\mathrm{s}}(\overrightarrow{\mathrm{p}}) \text { (creation operator of anti - fermion) }
\end{array}\right.
$$

These operators and their hermitic conjugates constitute fermion and anti-fermion operators. In taking to account the Pauli exclusion principle, the annihilation operator of a fermion $\hat{\mathrm{a}}_{\mathrm{s}}^{(-)}(\overrightarrow{\mathrm{p}})$ and the creation operator of fermion $\hat{\mathrm{a}}_{\mathrm{s}}^{\dagger(+)}(\overrightarrow{\mathrm{p}})$ take the following shapes[1,4]:

$$
\left\{\begin{array}{c}
\hat{\mathrm{c}} \equiv \hat{\mathrm{a}}_{\mathrm{s}}^{(-)}(\overrightarrow{\mathrm{p}})=\frac{1}{2}\left(\sigma_{\mathrm{x}}-\mathrm{i} \sigma_{\mathrm{y}}\right)=\left(\begin{array}{ll}
0 & 0 \\
1 & 0
\end{array}\right) \\
\hat{\mathrm{c}}^{\dagger} \equiv \hat{\mathrm{a}}_{\mathrm{s}}^{\dagger(-)}(\overrightarrow{\mathrm{p}})=\frac{1}{2}\left(\sigma_{\mathrm{x}}+\mathrm{i} \sigma_{\mathrm{y}}\right)=\left(\begin{array}{ll}
0 & 1 \\
0 & 0
\end{array}\right)
\end{array}\right.
$$

$\sigma_{\mathrm{x}}, \sigma_{\mathrm{y}}$ are the Pauli matrises. One interprets $\hat{\mathrm{n}}=\hat{\mathrm{c}}^{\dagger} \hat{\mathrm{c}}$ as an operator number of particles with eigenvalues 1 or 0 associed to the respective eigenfunctions $|1\rangle=\left(\begin{array}{l}1 \\ 0\end{array}\right)$ and $|0\rangle=\left(\begin{array}{l}0 \\ 1\end{array}\right)$.

\subsubsection{Anti-commutation Relations}

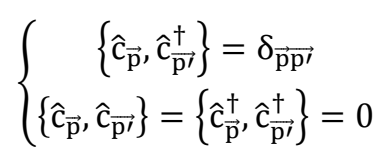

\subsubsection{Fock Space}

$$
\left\{\begin{array}{c}
\hat{\mathrm{c}}^{\dagger} \overrightarrow{\mathrm{p} \vec{\sigma}} \hat{\mathrm{c}}_{\overrightarrow{\mathrm{p}} \vec{\sigma}}|0\rangle=\mathrm{n}_{\overrightarrow{\mathrm{p}} \overrightarrow{\mathrm{\sigma}}}^{0}|0\rangle \\
\mathrm{n}_{\overrightarrow{\mathrm{p}} \vec{\sigma}}^{0}=\left\{\begin{array}{l}
1, \mathrm{p}<\mathrm{p}_{\mathrm{F}} \\
0, \mathrm{p}>\mathrm{p}_{\mathrm{F}}
\end{array}\right.
\end{array}\right.
$$




\subsubsection{Field Operators of Creation and Annihilation of} Electron of Impulsion $\vec{p}$ at the Position $\overrightarrow{\boldsymbol{r}}$

$$
\left\{\begin{array}{l}
\widehat{\psi}(\overrightarrow{\mathrm{r}}) \equiv \sum_{\overrightarrow{\mathrm{p}}} \hat{\mathrm{c}}_{\overrightarrow{\mathrm{p}}}\langle\mathrm{r} \mid \mathrm{p}\rangle=\sum_{\overrightarrow{\mathrm{p}}} \hat{\mathrm{c}}_{\overrightarrow{\mathrm{p}}} \varphi_{\overrightarrow{\mathrm{p}}}(\overrightarrow{\mathrm{r}}) \\
\widehat{\Psi}^{\dagger}(\overrightarrow{\mathrm{r}}) \equiv \sum_{\overrightarrow{\mathrm{p}}} \hat{\mathrm{c}}_{\overrightarrow{\mathrm{p}}}^{\dagger}\langle\mathrm{p} \mid \mathrm{r}\rangle=\sum_{\overrightarrow{\mathrm{p}}} \widehat{\mathrm{c}}_{\overrightarrow{\mathrm{p}}}^{\dagger} \varphi_{\overrightarrow{\mathrm{p}}}^{*}(\overrightarrow{\mathrm{r}})
\end{array}\right.
$$

\subsubsection{Anti-commutation Relations of Electron Field Operators}

$\left\{\begin{array}{c}\left\{\widehat{\psi}(\overrightarrow{\mathrm{r}}), \widehat{\psi}^{\dagger}\left(\overrightarrow{\mathrm{r}}^{\prime}\right)\right\}=\delta\left(\overrightarrow{\mathrm{r}}-\overrightarrow{\mathrm{r}}^{\prime}\right) \\ \left\{\widehat{\psi}(\overrightarrow{\mathrm{r}}), \widehat{\psi}\left(\overrightarrow{\mathrm{r}}^{\prime}\right)\right\}=\left\{\widehat{\psi}^{\dagger}(\overrightarrow{\mathrm{r}}), \widehat{\psi}^{\dagger}\left(\overrightarrow{\mathrm{r}}^{\prime}\right)\right\}=0\end{array}\right.$

\subsubsection{Hamiltonian Operator of Electrons System}

In second quantization, the Hamiltonian operator has the form

$$
\widehat{\mathrm{H}}=\int \mathrm{d} \overrightarrow{\mathrm{r}} \widehat{\psi}^{\dagger}(\overrightarrow{\mathrm{r}})\left[\frac{\hat{\mathrm{p}}^{2}}{2 \mathrm{~m}}+\widehat{\mathrm{V}}_{\text {piège }}\right] \widehat{\psi}(\overrightarrow{\mathrm{r}})+\frac{1}{2} \iint \mathrm{d} \overrightarrow{\mathrm{r}} \mathrm{d} \overrightarrow{\mathrm{r}}^{\prime} \widehat{\psi}^{\dagger}(\overrightarrow{\mathrm{r}}) \widehat{\psi}^{\dagger}\left(\overrightarrow{\mathrm{r}}^{\prime}\right) \widehat{\mathrm{V}}\left(\overrightarrow{\mathrm{r}}-\overrightarrow{\mathrm{r}}^{\prime}\right) \widehat{\psi}\left(\overrightarrow{\mathrm{r}}^{\prime}\right) \widehat{\psi}(\overrightarrow{\mathrm{r}})
$$

$\widehat{V}\left(\vec{r}-\vec{r}^{\prime}\right)$ is the long range Coulomb interaction.

\subsubsection{Equation of Movement (Temporal Evolution of Heisenberg Operator)}

$$
\hbar \frac{\partial \widehat{\psi}(\vec{r}, \mathrm{t})}{\partial \mathrm{t}} \cong\left(\frac{\widehat{p}^{2}}{2 \mathrm{~m}}+\widehat{\mathrm{V}}_{\text {piège }}+\int \mathrm{d} \vec{r}^{\prime} \widehat{\mathrm{V}}\left(\mathrm{r}^{\prime \prime}-\mathrm{r}^{\prime}\right)\left\langle\widehat{\psi}^{\dagger}\left(\mathrm{r}^{\prime}\right) \widehat{\psi}\left(\mathrm{r}^{\prime}\right)\right\rangle\right) \widehat{\psi}\left(\mathrm{r}^{\prime \prime}\right)-\int \mathrm{d} \overrightarrow{\mathrm{r}}^{\prime} \widehat{\psi}\left(\mathrm{r}^{\prime}\right) \widehat{\mathrm{V}}\left(\mathrm{r}^{\prime \prime}-\mathrm{r}^{\prime}\right)\left\langle\widehat{\psi}^{\dagger}\left(\mathrm{r}^{\prime}\right) \widehat{\psi}\left(\mathrm{r}^{\prime \prime}\right)\right\rangle
$$

\subsubsection{Dynamics of the System (Hartree-Fock Equation)}

$$
\varepsilon_{\mathrm{p}} \varphi_{\mathrm{p}}\left(\mathrm{r}^{\prime \prime}\right) \cong\left(\frac{\widehat{\mathrm{p}}^{2}}{2 \mathrm{~m}}+\widehat{\mathrm{V}}_{\text {piège }}+\frac{1}{\Omega} \int \mathrm{d} \overrightarrow{\mathrm{r}}^{\prime} \widehat{\mathrm{V}}\left(\mathrm{r}^{\prime \prime}-\mathrm{r}^{\prime}\right) \sum_{\mathrm{j}} \mathrm{n}_{\mathrm{j}} \varphi_{\mathrm{j}}^{*}\left(\mathrm{r}^{\prime}\right) \varphi_{\mathrm{j}}\left(\mathrm{r}^{\prime}\right)\right) \varphi_{\mathrm{p}}\left(\mathrm{r}^{\prime \prime}\right)-\int \mathrm{d} \overrightarrow{\mathrm{r}}^{\prime} \varphi_{\mathrm{p}}\left(\mathrm{r}^{\prime}\right) \widehat{\mathrm{V}}\left(\mathrm{r}^{\prime \prime}-\mathrm{r}^{\prime}\right) \sum_{\mathrm{j}} \mathrm{n}_{\mathrm{j}} \varphi_{\mathrm{j}}^{*}\left(\mathrm{r}^{\prime}\right) \varphi_{\mathrm{j}}\left(\mathrm{r}^{\prime \prime}\right)
$$

\subsubsection{Fundamental State Energy and Elementary Excitation Spectrum}

The fundamental state energy according to the Hamiltonian operator is

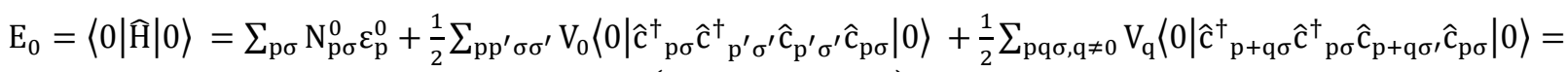

$$
\begin{aligned}
& \sum_{\mathrm{p} \sigma} \mathrm{N}_{\mathrm{p} \sigma}^{0} \varepsilon_{\mathrm{p}}^{0}+\frac{1}{2} \sum_{\mathrm{pp}^{\prime} \sigma \sigma^{\prime}} \mathrm{V}_{0} \mathrm{~N}_{\mathrm{p} \sigma}^{0}\left(\mathrm{~N}_{\mathrm{p}^{\prime} \sigma^{\prime}}^{0}-\delta_{\mathrm{p}^{\prime} \mathrm{p}} \delta_{\sigma^{\prime} \sigma}\right)+\sum_{\mathrm{pq} \sigma} \mathrm{V}_{\mathrm{q}} \mathrm{N}_{\mathrm{p}+\mathrm{q} \sigma}^{0} \mathrm{~N}_{\mathrm{p} \sigma}^{0}
\end{aligned}
$$

and the elementary excitations spectrum are

$$
\varepsilon_{\mathrm{p} \sigma}^{\mathrm{HFA}}=\frac{\partial \mathrm{E}_{0}^{\mathrm{HFA}}}{\partial \mathrm{N}_{\mathrm{p} \sigma}}=\varepsilon_{\mathrm{p}}^{0}+\sum_{\mathrm{p}^{\prime} \sigma^{\prime}} \mathrm{N}_{\mathrm{p}^{\prime} \sigma^{\prime}}^{0} \mathrm{~V}_{0}-\sum_{\mathrm{p}^{\prime} \sigma^{\prime}} \mathrm{N}_{\mathrm{p}^{\prime} \sigma^{\prime}}^{0} \mathrm{~V}_{\mathrm{p}^{\prime}-\mathrm{p}} \delta_{\sigma \sigma^{\prime}}
$$

\subsection{Properties of Ultra-cold Identical Ions of Spin Zero at High Density in rf Linear Paul Trap}

Ultra-cold trapped ions are used as a quantum memory for light $[19,20]$. We applied the microscopic theory to a gathering of identical ions of spin zero at high density confined in a radiofrequency linear Paul trap. The results of our investigation $[5,15]$ are the following:

\subsubsection{Fields of Spin Zero Ion and Anti-ion (Complex Scalar Field)}

$$
\left\{\begin{array}{l}
\phi(x)=\frac{1}{\sqrt{(2 \pi)^{3}}} \int \frac{d^{3} k}{\sqrt{2 k_{0}}}\left[a(\vec{k}) e^{i k x}+b^{*}(\vec{k}) e^{-i k x}\right] \\
\phi^{*}(x)=\frac{1}{\sqrt{(2 \pi)^{3}}} \int \frac{d^{3} k}{\sqrt{2 k_{0}}}\left[b(\vec{k}) e^{i k x}+a^{*}(\vec{k}) e^{-i k x}\right]
\end{array}\right.
$$

These fields are the expansion of the solutions of Klein Gordon equation which derives to a mass particle charged Lagrangian density.

\subsubsection{Spin Zero Ion and Anti-ion Operators}

In order to get ion and anti-ion operators, fields (31) are transformed into operators and the coefficients of the development are determined by inverse Fourier transforms as:

$$
\left\{\begin{array}{l}
\hat{a}_{\vec{k}}=\frac{1}{\sqrt{(2 \pi)^{3}}} \int \frac{d^{3} x}{\sqrt{2 \mathrm{k}_{0}}}\left[\frac{\partial \widehat{\phi}(\mathrm{x})}{\partial \mathrm{t}}-\mathrm{ik} \mathrm{k}_{0} \widehat{\phi}(\mathrm{x})\right] \mathrm{e}^{\mathrm{ikx}} \\
\widehat{\mathrm{b}}_{\overrightarrow{\mathrm{k}}}=\frac{1}{\sqrt{(2 \pi)^{3}}} \int \frac{\mathrm{d}^{3} \mathrm{x}}{\sqrt{2 \mathrm{k}_{0}}}\left[\frac{\partial \widehat{\Phi}^{*}(\mathrm{x})}{\partial \mathrm{t}}-\mathrm{ik}_{0} \widehat{\phi}^{*}(\mathrm{x})\right] \mathrm{e}^{\mathrm{ikx}}
\end{array}\right.
$$

$\hat{\mathrm{a}}_{\overrightarrow{\mathrm{k}}}, \widehat{\mathrm{b}}_{\overrightarrow{\mathrm{k}}}$ and their hermitic conjugates constitute ion and antiion operators.

\subsubsection{Commutation Relations of Ion and Anti-ion Operators}

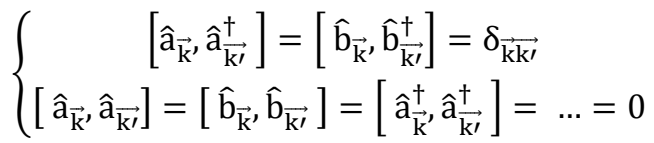

\subsubsection{Fock Space}

Let's consider the wave functions $\left|\ldots \mathrm{N}_{\overrightarrow{\mathrm{p}}}^{(+)} \ldots \mathrm{N}_{\overrightarrow{\mathrm{p}}}^{(-)} \ldots\right\rangle$ describing the stationary states, the wave function $\left|\mathrm{N}_{\overrightarrow{\mathrm{p}}}^{(+)}\right\rangle$corresponding to the state which in $\mathrm{N}_{\overrightarrow{\mathrm{p}}}^{(+)}$particles have a impulsion $\overrightarrow{\mathrm{p}}$ and a charge $\mathrm{Q}=\mathrm{e} \mathrm{N}_{\overrightarrow{\mathrm{p}}}^{(+)}$; the wave function $\left|\mathrm{N}_{\overrightarrow{\mathrm{p}}}^{(-)}\right\rangle$corresponding to the state which in $\mathrm{N}_{\overrightarrow{\mathrm{p}}}^{(-)}$particles have a impulsion $\overrightarrow{\mathrm{p}}$ and a charge $\mathrm{Q}=$ $-\mathrm{e} \mathrm{N}_{\overrightarrow{\mathrm{p}}}^{(-)}$. Thus, the Fock space becomes 


$$
\left|\mathrm{N}^{(+)}, \mathrm{N}^{(-)}\right\rangle=\frac{1}{\sqrt{\mathrm{N}^{(+)} ! \mathrm{N}^{(-) !}}}\left(\hat{\mathrm{a}}^{\dagger}\right)^{\mathrm{N}^{(+)}}\left(\hat{\mathrm{b}}^{\dagger}\right)^{\mathrm{N}^{(-)}}|0\rangle, \mathrm{N}^{(+)}, \mathrm{N}^{(-)}=0,1,2,3 \ldots
$$

with

$$
\left\langle\mathrm{N}^{(+)}, \mathrm{N}^{(-)} \mid \mathrm{M}^{(+)}, \mathrm{M}^{(-)}\right\rangle=\delta_{\mathrm{N}^{(+)} \mathrm{M}^{(+)}} \delta_{\mathrm{N}^{(-)} \mathrm{M}^{(-)}}
$$

and

$$
\begin{aligned}
& \left\{\begin{array}{l}
\hat{\mathrm{a}}_{\overrightarrow{\mathrm{p}}}^{\dagger}\left|\mathrm{N}_{\overrightarrow{\mathrm{p}}}^{(+)}, \mathrm{N}_{\overrightarrow{\mathrm{p}}}^{(-)}\right\rangle=\sqrt{\mathrm{N}_{\overrightarrow{\mathrm{p}}}^{(+)}+1}\left|\mathrm{~N}_{\overrightarrow{\mathrm{p}}}^{(+)}+1, \mathrm{~N}_{\overrightarrow{\mathrm{p}}}^{(-)}\right\rangle \\
\hat{\mathrm{b}}_{\overrightarrow{\mathrm{p}}}^{\dagger}\left|\mathrm{N}_{\overrightarrow{\mathrm{p}}}^{(+)}, \mathrm{N}_{\overrightarrow{\mathrm{p}}}^{(-)}\right\rangle=\sqrt{\mathrm{N}_{\overrightarrow{\mathrm{p}}}^{(-)}+1}\left|\mathrm{~N}_{\overrightarrow{\mathrm{p}}}^{(+)}, \mathrm{N}_{\overrightarrow{\mathrm{p}}}^{(-)}+1\right\rangle
\end{array}\right. \\
& \left\{\begin{array}{l}
\hat{\mathrm{a}}_{\overrightarrow{\mathrm{p}}}\left|\mathrm{N}_{\overrightarrow{\mathrm{p}}}^{(+)}, \mathrm{N}_{\overrightarrow{\mathrm{p}}}^{(-)}\right\rangle=\sqrt{\mathrm{N}_{\overrightarrow{\mathrm{p}}}^{(+)}}\left|\mathrm{N}_{\overrightarrow{\mathrm{p}}}^{(+)}-1, \mathrm{~N}_{\overrightarrow{\mathrm{p}}}^{(-)}\right\rangle \\
\widehat{\mathrm{b}}_{\overrightarrow{\mathrm{p}}}\left|\mathrm{N}_{\overrightarrow{\mathrm{p}}}^{(+)}, \mathrm{N}_{\overrightarrow{\mathrm{p}}}^{(-)}\right\rangle=\sqrt{\mathrm{N}_{\overrightarrow{\mathrm{p}}}^{(-)}}\left|\mathrm{N}_{\overrightarrow{\mathrm{p}}}^{(+)}, \mathrm{N}_{\overrightarrow{\mathrm{p}}}^{(-)}-1\right\rangle
\end{array}\right. \\
& \rho_{1}\left(\overrightarrow{\mathrm{r}}^{\prime}, \overrightarrow{\mathrm{r}}\right)=\left\langle\varphi(\mathrm{N})\left|\widehat{\psi}^{\dagger}(\overrightarrow{\mathrm{r}}) \widehat{\psi}\left(\overrightarrow{\mathrm{r}}^{\prime}\right)\right| \varphi(\mathrm{N})\right\rangle=\sum_{\overrightarrow{\mathrm{p}}} \mathrm{N}_{\overrightarrow{\mathrm{p}}}^{(+)} \varphi_{\overrightarrow{\mathrm{p}}}^{*}(\overrightarrow{\mathrm{r}}) \varphi_{\overrightarrow{\mathrm{p}}}\left(\overrightarrow{\mathrm{r}}^{\prime}\right)+\sum_{\overrightarrow{\mathrm{p}}} \mathrm{N}_{\overrightarrow{\mathrm{p}}}^{(-)} \varphi_{\overrightarrow{\mathrm{p}}}^{*}(\overrightarrow{\mathrm{r}}) \varphi_{\overrightarrow{\mathrm{p}}}\left(\overrightarrow{\mathrm{r}}^{\prime}\right)
\end{aligned}
$$

In a fundamental state, the one-body density matrix becomes

$$
\rho_{0}\left(\overrightarrow{\mathrm{r}}, \overrightarrow{\mathrm{r}}^{\prime}\right)=\mathrm{N}_{0}^{(+)} \varphi_{0}^{*}(\overrightarrow{\mathrm{r}}) \varphi_{0}\left(\overrightarrow{\mathrm{r}}^{\prime}\right)+\sum_{\overrightarrow{\mathrm{p}} \neq 0} \mathrm{~N}_{\overrightarrow{\mathrm{p}}}^{(+)} \varphi_{\overrightarrow{\mathrm{p}}}^{*}(\overrightarrow{\mathrm{r}}) \varphi_{\overrightarrow{\mathrm{p}}}\left(\overrightarrow{\mathrm{r}}^{\prime}\right)+\mathrm{N}_{0}^{(-)} \varphi_{0}^{*}(\overrightarrow{\mathrm{r}}) \varphi_{0}\left(\overrightarrow{\mathrm{r}}^{\prime}\right)+\sum_{\overrightarrow{\mathrm{p}} \neq 0} \mathrm{~N}_{\overrightarrow{\mathrm{p}}}^{(-)} \varphi_{\overrightarrow{\mathrm{p}}}^{*}(\overrightarrow{\mathrm{r}}) \varphi_{\overrightarrow{\mathrm{p}}}\left(\overrightarrow{\mathrm{r}}^{\prime}\right)
$$

Let's search for the limit of the one-body density matrix in a fundamental state when $\left|\vec{r}^{\prime}-\vec{r}\right|$ tends to zero

$$
\lim _{\left|\overrightarrow{\mathrm{r}}^{\prime}-\overrightarrow{\mathrm{r}}\right| \rightarrow 0} \rho_{0}^{( \pm)}\left(\overrightarrow{\mathrm{r}}, \overrightarrow{\mathrm{r}}^{\prime}\right)=\frac{1}{\Omega}\left[\mathrm{N}_{0}^{( \pm)}+\sum_{\overrightarrow{\mathrm{p}} \neq 0} \mathrm{~N}_{\overrightarrow{\mathrm{p}}}^{( \pm)}\right]
$$

It describes local correlations between the excited system particles.

The limit of one-body density matrix in a fundamental state when $\left[\vec{r}^{\prime}-\vec{r}\right]$ tends to $\infty$ is

$$
\begin{gathered}
\int \mathrm{d} \overrightarrow{\mathrm{r}}^{\prime} \rho_{1}\left(\overrightarrow{\mathrm{r}}^{\prime}, \overrightarrow{\mathrm{r}}\right) \varphi_{\overrightarrow{\mathrm{p}}}\left(\overrightarrow{\mathrm{r}}^{\prime}\right)=\int \mathrm{d} \overrightarrow{\mathrm{r}}^{\prime} \frac{1}{\Omega} \sum_{\overrightarrow{\mathrm{p}}}\left(\mathrm{N}_{\overrightarrow{\mathrm{p}}}^{(+)}+\mathrm{N}_{\overrightarrow{\mathrm{p}}}^{(-)}\right) \mathrm{e}^{\mathrm{i} \overrightarrow{\mathrm{p}} \cdot\left(\overrightarrow{\mathrm{r}}-\overrightarrow{\mathrm{r}}^{\prime}\right)} \varphi_{\overrightarrow{\mathrm{p}}}\left(\overrightarrow{\mathrm{r}}^{\prime}\right)=\int \mathrm{d} \overrightarrow{\mathrm{r}}^{\prime} \frac{1}{(2 \pi)^{3}} \int \mathrm{d} \overrightarrow{\mathrm{p}} \mathrm{e}^{\mathrm{i} \overrightarrow{\mathrm{p}} \cdot\left(\overrightarrow{\mathrm{r}}-\overrightarrow{\mathrm{r}}^{\prime}\right)}\left(\mathrm{N}_{\overrightarrow{\mathrm{p}}}^{(+)}+\mathrm{N}_{\overrightarrow{\mathrm{p}}}^{(-)}\right) \varphi_{\overrightarrow{\mathrm{p}}}\left(\overrightarrow{\mathrm{r}}^{\prime}\right)= \\
\int \mathrm{d} \overrightarrow{\mathrm{r}}^{\prime} \delta\left(\overrightarrow{\mathrm{r}}-\overrightarrow{\mathrm{r}}^{\prime}\right)\left(\mathrm{N}_{\overrightarrow{\mathrm{p}}}^{(+)}+\mathrm{N}_{\overrightarrow{\mathrm{p}}}^{(-)}\right) \varphi_{\overrightarrow{\mathrm{p}}}\left(\overrightarrow{\mathrm{r}}^{\prime}\right)=\mathrm{N}_{\overrightarrow{\mathrm{p}}}^{(+)} \varphi_{\overrightarrow{\mathrm{p}}}(\overrightarrow{\mathrm{r}})+\mathrm{N}_{\overrightarrow{\mathrm{p}}}^{(-)} \varphi_{\overrightarrow{\mathrm{p}}}(\overrightarrow{\mathrm{r}})
\end{gathered}
$$

It corresponds to the eigenfunction $\varphi_{\overrightarrow{\mathrm{p}}}(\overrightarrow{\mathrm{r}})$, with the largest eigenvalue $\mathrm{N}_{\overrightarrow{\mathrm{p}}}$.

In the case of wake interaction or a high density in the

trap, the fluctuations in the fundamental state are due to Coulomb interaction between charged particles. The Bogoliubov approximation leads for boson ions to

$$
\widehat{\psi}_{ \pm}(\vec{r}, t)=\sqrt{N_{0}^{( \pm)}} \varphi_{o}(\vec{r}, t)+\delta \widehat{\psi}_{ \pm}(\vec{r}, t)=\Phi_{ \pm}(\vec{r}, t)+\delta \widehat{\psi}_{ \pm}(\vec{r}, t)
$$

\subsubsection{Properties According to the Hamiltonian Operator}

In second quantization, the Hamiltonian operator has the form

$$
\widehat{\mathrm{H}}=\int d \overrightarrow{\mathrm{r}} \widehat{\psi}^{\dagger}(\overrightarrow{\mathrm{r}})\left[\frac{\widehat{\mathrm{p}}^{2}}{2 \mathrm{~m}}+\widehat{V}_{\text {piège }}\right] \widehat{\psi}(\overrightarrow{\mathrm{r}})+\frac{1}{2} \iint d \overrightarrow{\mathrm{r}} d \overrightarrow{\mathrm{r}}^{\prime} \widehat{\psi}^{\dagger}(\overrightarrow{\mathrm{r}}) \widehat{\Psi}^{\dagger}\left(\overrightarrow{\mathrm{r}}^{\prime}\right) \widehat{V}\left(\overrightarrow{\mathrm{r}}-\overrightarrow{\mathrm{r}}^{\prime}\right) \widehat{\psi}\left(\overrightarrow{\mathrm{r}}^{\prime}\right) \widehat{\psi}(\overrightarrow{\mathrm{r}})
$$

here $\widehat{V}\left(\vec{r}-\vec{r}^{\prime}\right)$ is the long range Coulomb interaction.

The temporal evolution of Heisenberg field operator leads to

$$
i \frac{\partial \widehat{\psi}\left(r^{\prime \prime}\right)}{\partial t}=-\left[\widehat{H}, \widehat{\psi}\left(r^{\prime \prime}\right)\right] \cong\left(\frac{\widehat{\Upsilon}^{2}}{2 m}+\widehat{V}_{\text {piège }}+\int d \vec{r}^{\prime} \widehat{V}\left(r^{\prime \prime}-r^{\prime}\right)\left\langle\widehat{\psi}^{\dagger}\left(r^{\prime}\right) \widehat{\psi}\left(r^{\prime}\right)\right\rangle\right) \widehat{\psi}\left(r^{\prime \prime}\right)+\int d \vec{r}^{\prime} \widehat{\psi}\left(r^{\prime}\right) \widehat{V}\left(r^{\prime \prime}-r^{\prime}\right)\left\langle\widehat{\psi}^{\dagger}\left(r^{\prime}\right) \widehat{\psi}\left(r^{\prime \prime}\right)\right\rangle
$$


and the dynamics of the system (Hartree-Fock Equation) is given by

$$
\varepsilon_{\mathrm{p}} \varphi_{\overrightarrow{\mathrm{p}}}\left(\mathrm{r}^{\prime \prime}\right) \cong\left(\frac{\widehat{\mathrm{p}}^{2}}{2 \mathrm{~m}}+\widehat{\mathrm{V}}_{\mathrm{piège}}+\int \mathrm{d} \overrightarrow{\mathrm{r}}^{\prime} \widehat{\mathrm{V}}\left(\mathrm{r}^{\prime \prime}-\mathrm{r}^{\prime}\right) \sum_{\mathrm{j}} \mathrm{N}_{\overrightarrow{\mathrm{j}}}^{(+)} \varphi_{\mathrm{j}}^{*}\left(\mathrm{r}^{\prime}\right) \varphi_{\vec{j}}\left(\mathrm{r}^{\prime}\right)\right) \varphi_{\overrightarrow{\mathrm{p}}}\left(\mathrm{r}^{\prime \prime}\right)+\int \mathrm{d} \overrightarrow{\mathrm{r}}^{\prime} \varphi_{\overrightarrow{\mathrm{p}}}\left(\mathrm{r}^{\prime}\right) \widehat{\mathrm{V}}\left(\mathrm{r}^{\prime \prime}-\mathrm{r}^{\prime}\right) \sum_{\overrightarrow{\mathrm{j}}} \mathrm{N}_{\overrightarrow{\mathrm{j}}}^{(+)} \varphi_{\overrightarrow{\mathrm{j}}}^{*}\left(\mathrm{r}^{\prime}\right) \varphi_{\mathrm{j}}\left(\mathrm{r}^{\prime \prime}\right)
$$

Equation (44) is the Hartree-Fock equation of zero spin positive charge boson in the radiofrequency linear Paul trap. For the negative charge boson, we have the equation

$$
\varepsilon_{\mathrm{p}} \varphi_{\overrightarrow{\mathrm{p}}}\left(\mathrm{r}^{\prime \prime}\right) \cong\left(\frac{\widehat{\mathrm{p}}^{2}}{2 \mathrm{~m}}+\widehat{V}_{\text {piège }}+\int \mathrm{d} \overrightarrow{\mathrm{r}}^{\prime} \widehat{V}\left(\mathrm{r}^{\prime \prime}-\mathrm{r}^{\prime}\right) \sum_{\mathrm{j}} \mathrm{N}_{\mathrm{j}}^{(-)} \varphi_{\vec{j}}^{*}\left(\mathrm{r}^{\prime}\right) \varphi_{\vec{j}}\left(\mathrm{r}^{\prime}\right)\right) \varphi_{\overrightarrow{\mathrm{p}}}\left(\mathrm{r}^{\prime \prime}\right)+\int \mathrm{d} \overrightarrow{\mathrm{r}}^{\prime} \varphi_{\overrightarrow{\mathrm{p}}}\left(\mathrm{r}^{\prime}\right) \widehat{V}\left(\mathrm{r}^{\prime \prime}-\mathrm{r}^{\prime}\right) \sum_{\overrightarrow{\mathrm{j}}} \mathrm{N}_{\overrightarrow{\mathrm{j}}}^{(-)} \varphi_{\vec{j}}^{*}\left(\mathrm{r}^{\prime}\right) \varphi_{\vec{j}}\left(\mathrm{r}^{\prime \prime}\right)
$$

The energy of the fundamental state of system of positive charge boson in the Paul trap is given by:

$$
\langle 0|\widehat{\mathrm{H}}| 0\rangle=\sum_{\mathrm{i}} \mathrm{N}_{\mathrm{i}}^{(+)} \varepsilon_{\mathrm{i}}^{0}+\frac{\left[\mathrm{N}_{0}^{(+)}\right]^{2}}{2}\left[\mathrm{~V}_{0}+\mathrm{V}_{\mathrm{q}}\right]+\frac{1}{2} \sum_{\mathrm{i}, \mathrm{i}^{\prime} \neq 0} \mathrm{~N}_{\mathrm{i}}^{(+)} \mathrm{N}_{\mathrm{i}^{\prime}}^{(+)}\left[\mathrm{V}_{0}+\mathrm{V}_{\mathrm{q}}\right]
$$

Similarly, one finds for the antiparticles

$$
\langle 0|\widehat{\mathrm{H}}| 0\rangle=\sum_{\mathrm{i}} \mathrm{N}_{\mathrm{i}}^{(-)} \varepsilon_{\mathrm{i}}^{0}+\frac{\left[\mathrm{N}_{0}^{(-)}\right]^{2}}{2}\left[\mathrm{~V}_{0}+\mathrm{V}_{\mathrm{q}}\right]+\frac{1}{2} \sum_{\mathrm{i}, \mathrm{i}^{\prime} \neq 0} \mathrm{~N}_{\mathrm{i}}^{(-)} \mathrm{N}_{\mathrm{i}}^{(-)}\left[\mathrm{V}_{0}+\mathrm{V}_{\mathrm{q}}\right]
$$

The elementary excitations spectrum is

$$
\begin{gathered}
\varepsilon_{\overrightarrow{\mathrm{p}}}=\frac{\mathrm{p}^{2}}{2 \mathrm{~m}}+\mathrm{V}_{\text {piège }}+\sum_{\overrightarrow{\mathrm{l}}} \mathrm{N}_{\overrightarrow{\mathrm{l}}}^{(+)} \int \mathrm{d}_{\mathrm{r}^{\prime}} \varphi_{\overrightarrow{\mathrm{l}}}^{*}\left(\mathrm{r}^{\prime}\right) \mathrm{V}\left(\mathrm{r}^{\prime \prime}-\mathrm{r}^{\prime}\right) \varphi_{\overrightarrow{\mathrm{l}}}\left(\mathrm{r}^{\prime}\right)+\sum_{\overrightarrow{\mathrm{j}}} \mathrm{N}_{\overrightarrow{\mathrm{j}}}^{(+)} \int \mathrm{d}^{\prime} \varphi_{\overrightarrow{\mathrm{j}}}^{*}\left(\mathrm{r}^{\prime}\right) \mathrm{V}\left(\mathrm{r}^{\prime \prime}-\mathrm{r}^{\prime}\right) \varphi_{\overrightarrow{\mathrm{j}}}\left(\mathrm{r}^{\prime \prime}\right)=\frac{\mathrm{p}^{2}}{2 \mathrm{~m}}+\mathrm{V}_{\text {piège }}+ \\
\mathrm{V}_{0} \sum_{\overrightarrow{\mathrm{l}}} \mathrm{N}_{\overrightarrow{\mathrm{l}}}^{(+)}+\mathrm{V}_{\mathrm{q}} \sum_{\overrightarrow{\mathrm{j}}} \mathrm{N}_{\overrightarrow{\mathrm{j}}}^{(+)}=\frac{\mathrm{p}^{2}}{2 \mathrm{~m}}+\mathrm{V}_{\text {piège }}+\sum_{\overrightarrow{\mathrm{p}}} \mathrm{N}_{\overrightarrow{\mathrm{p}}}^{(+)}\left[\mathrm{V}_{0}+\mathrm{V}_{\mathrm{q}}\right]
\end{gathered}
$$

\section{Conclusion}

At low temperature or at high density, the quantum effects of particles appear. The spin and the electric charge play a determining role in the quantum degeneration of identical particle system. The entire spin of a particle is responsible for the macroscopic occupation of a quantum state of a single particle, the Bose-Einstein condensate (BEC). Whereas the half-full spin is responsible for the Pauli exclusion among fermions, and leads to the Fermi sea. The short range interaction between particles allows to contact potential and leads to Gross-Pitaevskii equation, whereas the long range interaction leads to Hartree-Fock equation.

The use of real scalar field for describing neutral bosons of spin zero in the trap has given a good agreement with the experiment. It is the same with Dirac field in the study of electron gas in a metal. The complex scalar field has allowed us to determine the parameters of degeneration of the confined high density spin zero plasma as well as the energy, elementary excitations spectrum. The microscopic theory attributable to the quantum theory of fields has been successfully implemented in three different quantum systems in this paper.

\section{References}

[1] Weinberg S. (1995). The quantum theory of fields. Cambridge university press, volume I and II. New York.

[2] Brantut J. P., Manipulation d'atomes froids dans des potentiels lumineux, thèse de doctorat, 2009.

[3] Dieckmann K., Spreeuw R. J. C, Weidemüller M. \& Walraven J. T. M. (1998). Two-dimensional magneto-optical trap as a source of slow atoms. Phys. Rev. A, vol. 58, no 5, pages 389163895, Nov.

[4] Vittel C. (1967). Théorie Quantique du Solide. Dunod, Paris.

[5] Tshizanga (2011). Dégénérescence quantique d'un système de bosons chargés identiques de spin zéro dans un piège de Paul. Thèse de doctorat, Université Nationale Pédagogique de Kinshasa. Tel-00760356, Version1- 6 Déc. 2012.

[6] Shaposhnikov M., (2007). Champs Quantiques Relativistes, EPF, Lausanne.

[7] Pagels H., (1985). L'univers quantique. InterEditions, Paris.

[8] Delamotte B. (2005). Introduction à la théorie quantique des champs. www.Iptmc.jussieu.fr.

[9] Nozières P. and Pines D. (1999). The theory of quantum liquids. Perseus books, Cambridge.

[10] Pethick C. J., Smith H. (2002). Bose-Einstein Condensation in Dilute Gases. Cambridge University Press. UK.

[11] Govaerts J., (1995), L'interaction électrofaible: une fenêtre sur la physique au-delà du Modèle Standard, Procedings of the Ecole Internationale Joliot-Curie de Physique Nucléaire, Noyaux en Collisions", Maubuisson (France), 11 - 16 Septembre 1995, éd. Y. Abgrall, pp. 333-416.

[12] Caradoc-Davies B. M. (2000). Vortex Dynamics in Bose-Einstein Condensates, These, University of Otago, 200 pages (2000).

[13] Tshizanga (2016), Hartree-Fock Equation for a Non-neutral Plasma of Spin Zero Ions in a Paul Trap; American Journal of Physics and Applications. Vol. 4, No. 3, 2016, pp. 71-77. doi: 10.11648/j.ajpa.20160403.11.

[14] Dalfovo F., Giorgini S., Pitaevskii L. P. and Stringari S. (1999), Theory of Bose-Einstein condensation in trapped gases. Reviews of Modern Physics, Vol. 71, No. 3, April, pp 463-512. 
[15] Tshizanga F. M., Badibanga P. M. and Ntampaka B. B. (2014), An investigation into the Parameters of Quantum Degeneration of an Ultra Cold Non-Neutre Plasma of identical Ions of Zero Spin in a Paul Trap. International Journal of Measurement Technologies and Instrumentation Engineering, 4 (1), 51-70. January-March 2014.

[16] Pei-Lin You, Large-Scale Bose-Einstein condensation in a vapor of cesium atoms at normal temperature $(\mathrm{T}=353 \mathrm{~K}), \mathrm{J}$ Material Sci Eng 5: 276 (2016). Doi: 10.4172/21690022.1000276

[17] Pei-Lin Yu. Large-Scale Bose-Einstein Condensation in an Atomic Gas by Applying an Electric Field. American Journal of Modern Physics. Vol. 7. No 4, 2018, pp. 121-130. doi: 10.11648/j.ajmp.20180704.11.

[18] Pei-Lin You, Bose-Einstein condensation in a vapor of sodium atoms in an electric field, Physica B: condensed matter physics 491(2016) 8492, http://dx.doi.org/10.1016/j.physb.2016.03.017.

[19] Mortensen A. Aspects of Ion Coulomb Crystal based Quantum Memory for Light. PhD thesis; 2005.

[20] Dubess R. Réalisation, étude et exploitation d'ensembles d'ions refroidis par laser stockés dans des pièges microfabriqués pour l'information quantique. These de doctorat. 2011. 